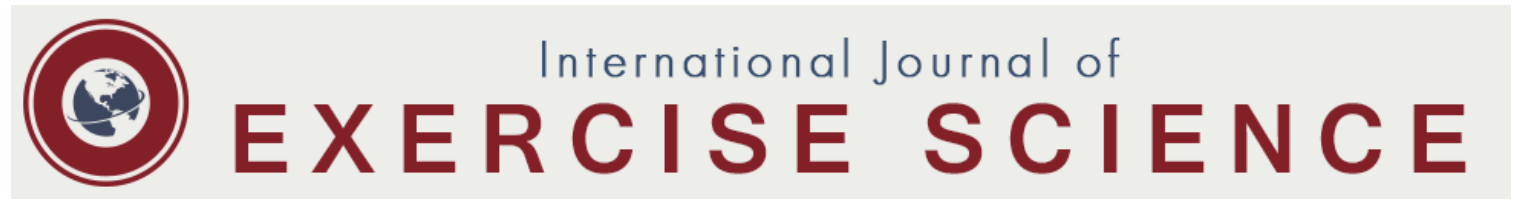

Original Research

\title{
Irisin and Fibronectin Type III Domain-Containing 5 Responses to Exercise in Different Environmental Conditions
}

\author{
MATTHEW P. BUBAK ${ }^{\dagger}$, MATTHEW W.S. HEESCH', ROBERT J. SHUTE ${ }^{\dagger}$, \\ NICHOLAS E. DINAN ${ }^{\dagger}$, TERENCE L. LAURSEN ${ }^{\dagger}$, D. TAYLOR LA SALLE ${ }^{\dagger}$, and \\ DUSTIN R. SLIVKA $\ddagger$
}

Health, Physical Education, and Recreation, University of Nebraska at Omaha, Omaha, Nebraska, USA

†Denotes graduate student author, ‡Denotes professional author

\begin{abstract}
International Journal of Exercise Science 10(5): 666-680, 2017. Fibronectin type III domain-containing 5 (FNDC5) is a skeletal muscle membrane-bound precursor to the myokine irisin. Irisin is involved in stimulating adipose tissue to become more metabolically active in order to produce heat. The purpose of this study was to determine the effects of exercise in a hot $\left(33^{\circ} \mathrm{C}\right)$, cold $\left(7^{\circ} \mathrm{C}\right)$, and room temperature $\left(\mathrm{RT}, 20^{\circ} \mathrm{C}\right)$ environment on the skeletal muscle gene expression of FNDC5 and the plasma concentrations of irisin. Twelve recreationally trained males completed three separate, $1 \mathrm{~h}$ cycling bouts at $60 \%$ of $\mathrm{W}_{\max }$ in a hot, cold, and RT environment followed by three hours of recovery at room temperature. Blood samples were taken from the antecubital vein and muscle biopsies were taken from the vastus lateralis pre-, post-, and $3 \mathrm{~h}$ post-exercise. Plasma concentrations of irisin did not change from pre- $(9.23 \pm 2.68$ $\left.\mathrm{pg} \cdot \mathrm{mL}^{-1}\right)$ to post-exercise $(9.6 \pm 0.2 \mathrm{pg} \mathrm{mL}-1, \mathrm{p}=0.068)$, but did decrease from post-exercise to $3 \mathrm{~h}$ post-exercise $\left(8.9 \pm 0.5 \mathrm{pg} \mathrm{mL}^{-1}, \mathrm{p}=0.047\right)$ regardless of temperature. However, when plasma volume shifts were considered, no differences were found in irisin $(p=0.086)$. There were no significant differences between trials for irisin plasma concentrations $(p>0.05)$. No significant differences in FNDC5 were observed between the hot, cold, or RT or pre-, post-, or $3 \mathrm{~h}$ postexercise time points $(\mathrm{p}>0.05)$. These data indicate that the temperature in which exercise takes place does not influence FNDC5 transcription or circulating irisin in a human model.
\end{abstract}

KEY WORDS: Brown fat, gene expression, hot, cold, FNDC5, epinephrine, norepinephrine

\section{INTRODUCTION}

In the United States a growing number of individuals suffer from metabolic and mitochondrial diseases, such as diabetes and obesity, which decrease life expectancy and quality of life $(5,23$, $27,40)$. Exercise mitigates the negative effects of metabolic diseases and releases a cascade of hormones, such as epinephrine and norepinephrine, that influence physiological alterations 
including: increased metabolism, increased glucose sensitivity, increased mitochondrial biogenesis and function $(2,14,19)$, by reversing the effects of a sedentary lifestyle (12). The release of certain catecholamines, cytokines, and myokines are responsible for signaling physiological adaptations during acute exercise bouts or training programs $(16,28)$. The myriad of signaling mechanisms that can occur during a typical exercise response may be altered when undertaken in different environmental temperatures (35). During exercise, physiological mechanisms such as metabolism and mitochondrial function can be blunted, enhanced, or unaffected by environmental temperature exposure. Cold stimulated, metabolically active, brown adipose tissue (BAT) has been discovered in humans $(32,39)$, and research has focused on the underlying mechanisms and metabolic implications. Specifically, the mechanism and dynamics by which irisin, a myokine, contributes to the "browning" of white adipose tissue is of particular interest due to the potential positive impact on health related outcomes, such as increased metabolic expenditure.

Irisin is a cytokine released from the muscle, thus termed a myokine, which is involved in the "browning" of white adipose tissue (WAT) (2). "Browning" WAT is characterized by an increase in mitochondria density and metabolic function in both brown and white adipose tissue, therefore increasing the overall energy expenditure (2). Irisin was discovered in 2012 (2) and has become a popular research topic due to the discovery of BAT in humans. BAT has non-shivering thermogenic capabilities due to an increase in mitochondrial density and thus has increased metabolic activity compared to WAT (2). During non-shivering thermogenesis, epinephrine and norepinephrine are secreted into the bloodstream to maintain metabolic homeostasis $(9,24)$. It is hypothesized that epinephrine and norepinephrine are upstream stimulators of irisin production during maximal and submaximal cycle ergometer endurance exercise (20). The increased metabolic energy produced during non-shivering thermogenesis is dissipated as heat to maintain core body temperature and increases overall energy expenditure (5). If this "browning" of WAT in humans is similar to that of animal models, the proposed mechanism of increased irisin would increase BAT in humans. This adaptation is positively related to health and may lead to protection against chronic diseases such as obesity and diabetes (23).

Fibronectin type III domain-containing 5 (FNDC5) is the protein precursor that is proteolytically cleaved to produce irisin (2). FNDC5 is a single peptide transmembrane protein inserted into the cellular membrane of skeletal muscle and WAT $(10,31)$ and was discovered in 2002 prior to irisin (8). Due to BAT's increased mitochondria density, peroxisome proliferator-activated receptor gamma coactivator 1 alpha (PGC-1 $\alpha$ ), which is highly expressed in tissues with high mitochondrial density, has been thought to regulate the expression of FNDC5, thus regulating irisin. PGC-1 $\alpha$ would therefore be required for proper function of BAT $(2,41)$. PGC- $1 \alpha$ is often referred to as the master regulator of mitochondrial biogenesis and contributes to the regulation of metabolism (22). Thus, the browning of white adipose tissue is suggested to be dependent on PGC-1 $\alpha$ and therefore plays a significant role in the regulation of irisin and BAT. 
Exercising in cold environmental temperatures increases PGC-1 $\alpha$ mRNA above that of exercise in room temperature control conditions and thus cold temperature interventions would be expected to stimulate mitochondrial biogenesis (35) and possibly up-regulate FNDC5. This increased irisin, derived from increased expression of FNDC5, is thought to drive BAT-like development, or "browning" of WAT and thermogenesis during cold exposure $(2,27)$. Similarly, irisin levels increase in subjects exposed to cold environmental temperatures due to the increased non-shivering thermodynamics (20). It has been hypothesized that exercise mimics the body's shivering thermogenesis by increasing plasma concentrations of irisin and expression of FNDC5 and PGC-1 $\alpha$ during cold environmental exposure (2). Inversely of cold exposure, exercising in a heated environment inhibits the expression of PGC-1 $\alpha$ mRNA (35), and may be expected to subsequently reduce FDNC5 expression and plasma irisin concentrations. However, previous research has not described this response of FNDC5 to exercise in the hot or cold compared to a room temperature control environment.

The purpose of this experimental study is to determine the effects of endurance exercise in a hot $\left(33^{\circ} \mathrm{C}\right)$, cold $\left(7^{\circ} \mathrm{C}\right)$, and room temperature $\left(20^{\circ} \mathrm{C}\right)$ environment on the plasma concentrations of irisin, epinephrine, and norepinephrine as well as the skeletal muscle expression of the gene FNDC5 in recreationally active males. Based on previous findings of PGC-1 $\alpha$ mRNA expression (35), we hypothesize that exercise in a cold environment will elicit greater circulating irisin and skeletal muscle expression of FNDC5 when compared to exercise in a room temperature environment and that irisin concentrations and expression of FNDC5 will be blunted following exercise in the hot environment compared to room temperature.

\section{METHODS}

\section{Participants}

Twelve healthy, recreationally active males (age: $25 \pm 4$, height: $178 \pm 5 \mathrm{~cm}$, weight: $79.2 \pm 12.8$ $\mathrm{kg}$, body fat: $14.5 \pm 3.5 \%, \mathrm{VO}_{2 \text { peak: }} 4.29 \pm 0.82 \mathrm{~L} \cdot \mathrm{min}^{-1}$, Power at $\mathrm{VO}_{2 \text { peak: }} 276 \pm 29$ ) were recruited from the University of Nebraska at Omaha and the surrounding area. The subjects were absent of any medical contraindications and apparently healthy individuals. All participants were informed of the risks associated with the study. The participants freely and willingly signed an Institutional Review Board (IRB) approved informed consent document before participation and abided by the Declaration of Helsinki (42).

\section{Protocol}

Body composition was measured using hydrodensitometry by using a custom electronic load cell based system (Exertech, Dresbach, MN). Residual lung and gastrointestinal volume were estimated using previously developed prediction equations (37) and used to correct body density values. Body density was then converted to a body fat percentage using the Siri equation (34).

Participants performed a maximal cycling aerobic capacity test $\left(\mathrm{VO}_{2 \text { peak }}\right)$ using a flow and gas calibrated metabolic cart (Parvomedics Inc., Salt Lake City, Utah) until voluntary exhaustion. Expired gases were measured continuously and averaged in 15 second intervals. The exercise 
protocol consisted of a graded test completed on an electronically braked Velotron Cycle ergometer (RacerMate Inc., Seattle, WA). The beginning stage workload was 95 watts (W) and increased every three minutes by $35 \mathrm{~W}$ until voluntary exhaustion. The resistance for the subsequent experimental trials was set to $60 \%$ of the power output at $\mathrm{VO}_{2 \text { peak. }}$.

Trials consisted of cycling at $60 \%$ of the subject's workload associated with $\mathrm{VO}_{2 \text { peak }}$ for one hour in a cold $\left(7^{\circ} \mathrm{C}, 60 \%\right.$ humidity), room temperature $\left(20^{\circ} \mathrm{C}, 60 \%\right.$ humidity), and hot $\left(33^{\circ} \mathrm{C}\right.$, $60 \%$ humidity) environment and three hours recovery at room temperature. These temperatures have been used previously and allow the participants to complete the cycling bout without an unsafe rise in core body temperature (35). Subjects had no exposure to the environmental conditions prior to the exercise bout and started exercise immediately upon entering the chamber and mounting the cycle ergometer. Each participant completed all three trials in a randomized and counter-balanced order. Experimental trials were separated by at least four days and conducted in a temperature and humidity controlled environmental chamber (Darwin, St. Louis, MO). Subjects kept a 48 hour exercise record and a 24 hour dietary record to replicate prior to all trials. Subjects were asked to abstain from exercise 24 hours prior to each trial and arrive in the laboratory after an 8 hour fast. During exercise the subjects consumed $500 \mathrm{~mL}$ of water over the course of each trial with approximately $125 \mathrm{~mL}$ being consumed every $15 \mathrm{~min}$. After exercise, subjects toweled off and recovered in the supine position for three hours in a separate room, at room temperature and water was consumed ad libitum and this amount was repeated during subsequent trials.

Gases were collected via metabolic cart (Parvomedics Inc., Sandy, Utah) for five minutes at four separate occasions over the course of each exercise bout: minutes 10-15, 25-30, 40-45, and 55-60. Gases were collected on three separate occasions during recovery: minutes 25-30, 85-90, and 145-150. The last three minutes of the 5 minute gas collection period were averaged to represent that time point. Additionally, the four exercise collection periods were averaged to represent the exercise condition and the three collection periods were averaged to represent the recovery condition.

Six subjects were utilized for monitoring core temperature due to technical problems with data recording. The participants ingested a Jonah Core Body Temperature Capsule (JCBC; Hidalgo Limited, Cambridge, UK) with $150 \mathrm{~mL}$ of water and a Fiber One granola bar (General Mills, Minneapolis, MN: 140 calories, $2 \mathrm{~g}$ of saturated fat, $90 \mathrm{mg}$ of sodium, $10 \mathrm{~g}$ of sugar, and $9 \mathrm{~g}$ of fiber, and $2 \mathrm{~g}$ of protein) to ensure proper digestion of the core temperature pill into the gastrointestinal tract. The core body temperature telemetry capsule data and skin temperature infrared thermistor data from the chest were logged via EQO2 LifeMonitor Sensor Electronics Module (SEM; Hidalgo Limited) that also recorded heart rate at 5 second intervals.

Three muscle biopsies were performed during each experimental visit via percutaneous needle biopsy technique with the aid of suction (1). Approximately five minutes prior to each muscle biopsy, approximately $3 \mathrm{~mL}$ of lidocaine was utilized as a local anesthetic to numb the biopsy area. The muscle samples were taken from the vastus lateralis pre-, post-, and three hours postexercise on the same leg for a given trial. Post-exercise muscle biopsies occurred $16.0 \pm 2.54$ 
min after completion of the exercise trial and the three hours post-exercise biopsy was time so that the muscle would be extracted approximately $3 \mathrm{~h}$ after exercise ( $2 \mathrm{~h} 57 \mathrm{~min} \pm 4 \mathrm{~min})$. The initial biopsy was taken approximately $12 \mathrm{~cm}$ proximal to the patella from the belly of the vastus lateralis. Subsequent biopsies were taken two centimeters proximal to the previous incision to avoid substantial damage to the muscle. The leg biopsied was randomized and alternated for the subsequent trial visits to account of leg dominance and leg bias during cycling. The samples were immediately cleared of excess blood and connective tissue and placed in RNA later solution (Ambion, Grand Island, NY). The samples were then stored at -80 ${ }^{\circ} \mathrm{C}$ for later analysis. The mean time from muscle biopsy to submersion of samples in RNA later was 2 minutes 36 seconds \pm 1 minute 24 seconds.

Muscle biopsy samples were analyzed for the gene expression of FNDC5, the precursor to irisin. Approximately $36.1 \pm 10.8 \mathrm{mg}$ of skeletal muscle was homogenized using an electric homogenizer (PowerGen 125, Fischer Scientific, Pittsburg, PA) in 800 ul of Trizol Reagent (Invitrogen, Carlsbad, CA). The RNA was further cleaned using an RNeasy mini kit (Qiagen, Valencia, CA). The RNA was reverse transcribed via SuperSript III First-Strand Synthesis kit (Invitogen, Carlsbad, CA) per manufacturer instruction to produce cDNA. The cDNA served as a template to run the quantitative real-time polymerase chain reaction (qRT-PCR) using Stratagene Mx30005P (Agilent Technologies, Santa Clara, CA). Parameters were set according to manufacturer's instructions at one cycle at $95{ }^{\circ} \mathrm{C}$ for three minutes, then 40 cycles at $95{ }^{\circ} \mathrm{C}$ for five seconds and $60{ }^{\circ} \mathrm{C}$ for 20 seconds (Agilent Technologies). Probes and primers were designed through Integrated DNA Technologies (Coralville, IA). An average of $951.7 \pm 547 \mathrm{ng}$ of mRNA was obtained and $0.625 \mathrm{ng} \cdot \mu \mathrm{l}^{-1}$ of cDNA was added for each $20 \mu \mathrm{l}$ PCR reaction. RNA integrity was measured using an Agilent 2100 bioanalyzer (Agilent Technologies) which yielded a RNA integrity number of $8.1 \pm 0.7$, indicating high quality mRNA. FNDC5 mRNA is expressed relative to stable reference genes using the $2^{-\mathrm{a} C t}$ method (21). Qbase+ geNorm software (Biogazelle, Gent, Belgium) was utilized to determine the stability and optimal number of reference genes. The candidate reference genes used were: RPS18, $\beta$-actin, cyclophillin, GAPDH, and B2M. These reference genes have demonstrated stability in response to temperature and exercise. Candidate genes were selected based on stability of each individual participant. If multiple genes were selected for a given participant, the geometric mean of these stable references genes was calculated. Gene expression was measured post-exercise and $3 \mathrm{~h}$ post-exercise for FNDC5 and normalized to the geometric mean of the stable reference genes and the pre-exercise condition for each participant. One participant was omitted from the gene expression analysis due to low sample quality.

Approximately $6 \mathrm{~mL}$ of blood was drawn from the antecubital vein into an $8 \mathrm{~mL}$ EDTA vacutainer tube (Greiner Bio-One, Monroe, NC), pre-, post-, and three hours post-exercise. Whole blood was immediately analyzed for hemoglobin concentration (HemoCue $\mathrm{Hb}_{201^{+}}$ Analyzer, Ängelholm, Sweden) and hematocrit (Zipocrit, LW Scientific Inc, Lawrenceville, Georgia) and utilized to correct the concentration of target hormones due to the known plasma shifts that occur with heat exposure and exercise, despite water ingestion (6). The remaining blood sample was centrifuged (Thermo Scientific, Waltham, MA) at $1000 \mathrm{RPM}$ at $7^{\circ} \mathrm{C}$ for 10 minutes and the plasma was aliquoted and frozen for later analysis. One participant was 
omitted from the blood sample analysis due to technical issues with hematocrit and hemoglobin measurements.

The plasma samples were analyzed for irisin using an Irisin Competitive Enzyme-linked immunosorbent assay (ELISA) kit (BioVendor, Brno, Czech Republic) according to the manufacturer instructions. Collected plasma was diluted with diluent $1 \mathrm{X}$ to a 1:4 ratio. The plate was then measured for protein content at $450 \mathrm{~nm}$ in a spectrophotometric plate reader (Fischer Scientific, Pittsburg, PA).

Epinephrine and norepinephrine concentrations were determined using an Epinephrine/Norepinephrine ELISA kit (Abnova, Taipei City, Taiwan) in microplate format. The procedure was completed according to manufacturer protocol. The sample absorbance is quantified using a spectrophotometric plate reader (Fischer Scientific, Pittsburg, PA) at $450 \mathrm{~nm}$ against a reference wavelength between $620 \mathrm{~nm}$ and $650 \mathrm{~nm}$. All ELISAs were completed in duplicate. The coefficient of variance for duplicate samples was $4.3 \pm 4.0 \%$.

\section{Statistical Analysis}

$\mathrm{VO}_{2}$, core temperature, environmental conditions, heart rate, FNDC5 gene expression, and plasma levels of irisin, epinephrine, and norepinephrine were analyzed using a (time $x$ condition) repeated measures two-way ANOVA. If significance was detected, a Fishers protected LSD post hoc analysis was performed to detect where differences occurred. The significance level was set at $p<0.05$. All data were analyzed using Statistical Package for Social Sciences software (SPSS 23.0, Chicago, IL) and reported as mean \pm SE.

\section{RESULTS}

During exercise, core temperature was greater in the hot compared to the cold and RT from the 50 minute time point of exercise to the end of exercise $(p<0.05$, Figure 1$)$. During recovery, core temperature was higher in the hot compared to the RT $(p=0.001)$ and cold $(p=0.027)$ during the first hour but was not different thereafter $(p>0.05$, Figure 1). During exercise, skin temperature was significantly higher in the hot $\left(36.4 \pm 0.5^{\circ} \mathrm{C}\right)$ compared to the RT (32.4 \pm 2.1 $\left.{ }^{\circ} \mathrm{C}\right)$ and cold $\left(27.8 \pm 1.1^{\circ} \mathrm{C}\right)$, and RT was higher than the cold $(\mathrm{p}<0.001)$. During recovery, skin temperature was higher in the RT $\left(34.5 \pm 0.8^{\circ} \mathrm{C}\right)$ compared to the cold $\left(33.5 \pm 0.8^{\circ} \mathrm{C}, \mathrm{p}=0.025\right)$ and no difference was observed from the hot $\left(34.3 \pm 1.1^{\circ} \mathrm{C}\right)$.

During exercise absolute oxygen consumption $\left(\mathrm{VO}_{2}\right)$ was greater in the hot $\left(3.02 \pm 0.46 \mathrm{~L} \cdot \mathrm{min}^{-}\right.$ $\left.{ }^{1}\right)$ compared to RT $\left(2.80 \pm 0.38 \mathrm{~L} \cdot \mathrm{min}^{-1}\right)$ and RT was greater than the cold $\left(2.67 \pm 0.36 \mathrm{~L} \cdot \mathrm{min}^{-1}\right.$, $\mathrm{p}=0.002)$. During recovery, there were no differences in oxygen consumption between the three conditions $\left(0.35 \pm 0.06 \mathrm{~L} \cdot \mathrm{min}^{-1}, 0.34 \pm 0.06 \mathrm{~L} \cdot \mathrm{min}^{-1}, 0.34 \pm 0.05 \mathrm{~L} \cdot \mathrm{min}^{-1}, \mathrm{p}=0.372\right)$. Heart rate (HR) was higher in the hot $(38.7 \pm 6.4 \mathrm{bpm})$ compared to RT $(155 \pm 12 \mathrm{bpm})$ and the cold $(152 \pm 9)$ during exercise $(p<0.001)$. There was no difference in HR during exercise between cold and RT $(p=0.238)$. There were no differences in HR during recovery between conditions $(\mathrm{p}>0.05)$. 


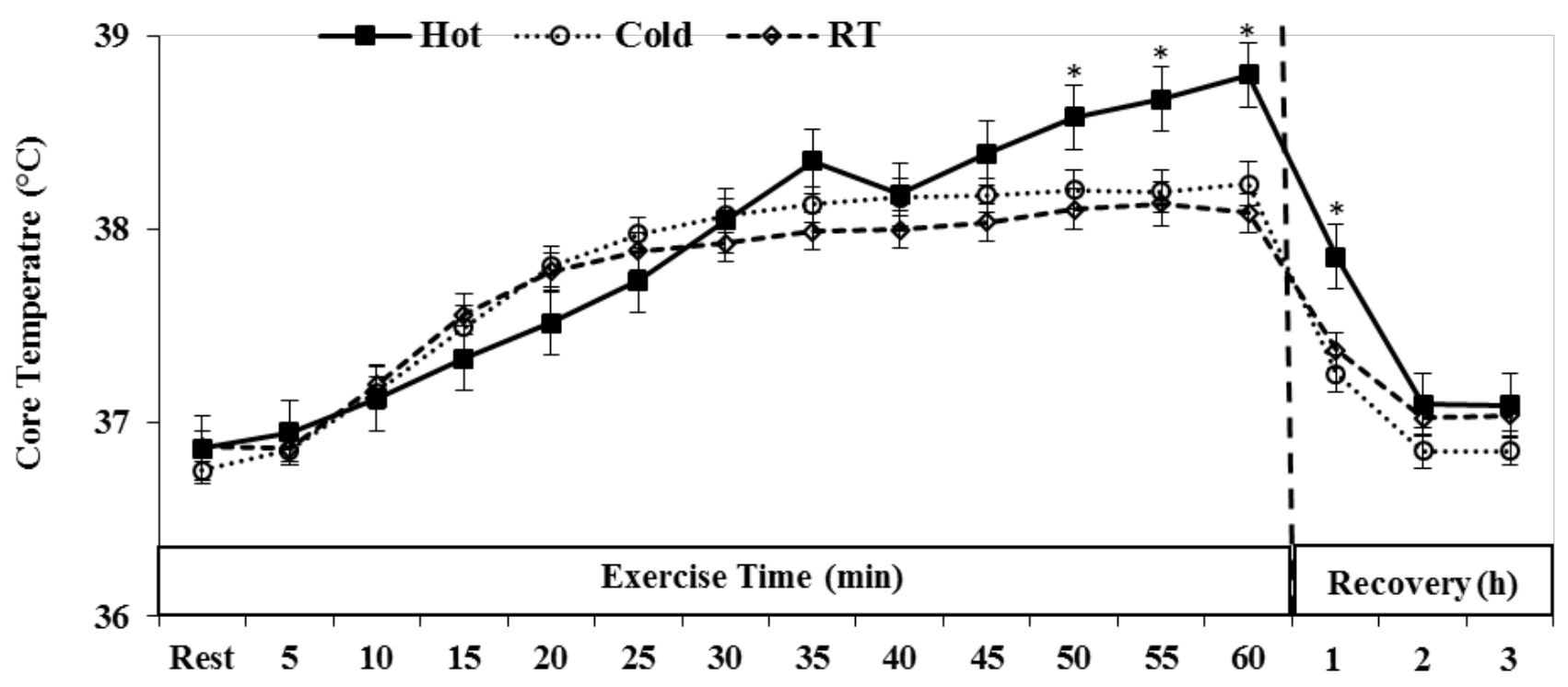

Figure 1. Core temperature during exercise and recovery where ${ }^{*} p<0.05$ is from RT and cold. Data are mean \pm SE.

No differences were observed for irisin plasma concentrations occurred from pre- to postexercise $(p=0.068$, Figure $2 A)$. However, there was a significant decrease from post-exercise to resting levels at $3 \mathrm{~h}$ post-exercise $(\mathrm{p}=0.047$, Figure $2 \mathrm{~A}$ ) when not considering plasma volume shifts regardless of temperature. However, when plasma volume shifts were considered, there was no difference between pre- and post-exercise $(p=0.984$, Figure $2 B)$ and only a trend toward decreased irisin plasma concentration was observed from post-exercise to $3 \mathrm{~h}$ postexercise $(p=0.086$, Figure $2 B)$. There were no differences between environmental conditions ( $p$ $=0.39$ ).
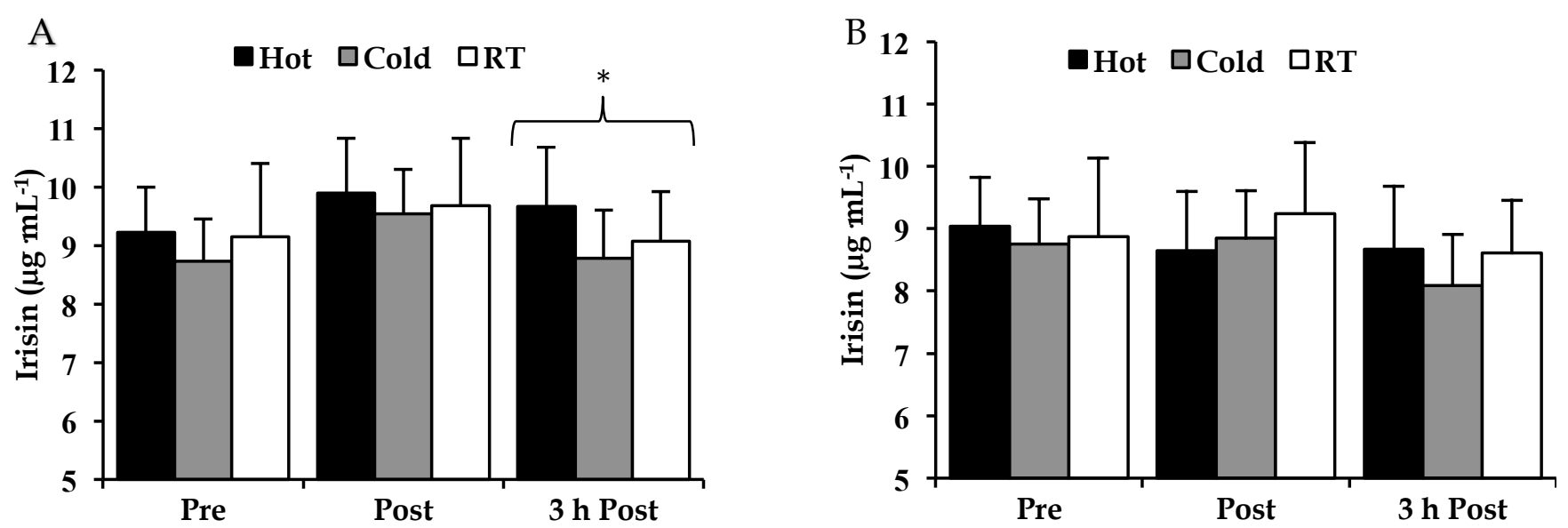

Figure 2. Irisin plasma concentrations without plasma volume shift corrections (A) and after correcting for plasma volume shifts $(B),{ }^{*} p<0.05$ from post-exercise. Data are mean $\pm S E$. 
Epinephrine plasma concentrations were significantly increased post-exercise from preexercise in all conditions $(p<0.05$, Figure 3A). Epinephrine decreased in hot and cold from post-exercise to $3 \mathrm{~h}$ post-exercise ( $\mathrm{p}<0.05$, Figure $3 \mathrm{~A})$. Epinephrine was higher post-exercise in the hot compared to RT ( $<<0.05$, Figure 3A) and a trend in increased epinephrine plasma concentration in hot occurred compared to the cold $(\mathrm{p}<0.10$, Figure $3 \mathrm{~A})$. At $3 \mathrm{~h}$ post-exercise, epinephrine in the cold trial was higher than hot $(\mathrm{p}<0.05$, Figure $3 \mathrm{~A})$. At $3 \mathrm{~h}$ post-exercise, epinephrine was higher in the cold and RT compared to pre-exercise ( $p<0.05$, Figure 3A). Similar results were observed for epinephrine when plasma concentrations were corrected for plasma volume shifts except that epinephrine in the RT condition only trended to be higher at $3 \mathrm{~h}$ post- compared to pre-exercise ( $p=0.052$, Figure $3 \mathrm{~B})$.
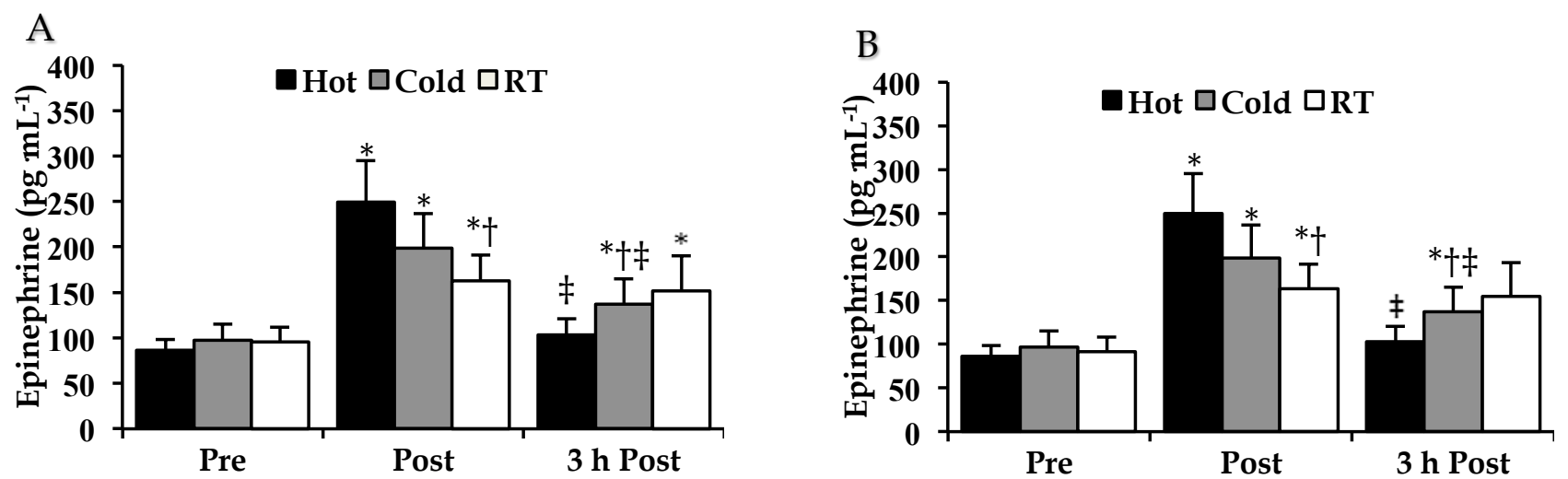

Figure 3. Epinephrine plasma concentrations without plasma volume shift corrections (A) and after correcting for plasma volume shifts $(B),{ }^{*} p<0.05$ from pre-exercise, $\uparrow p<0.05$ from hot, $\ddagger p<0.05$ from post-exercise. Data are mean \pm SE.
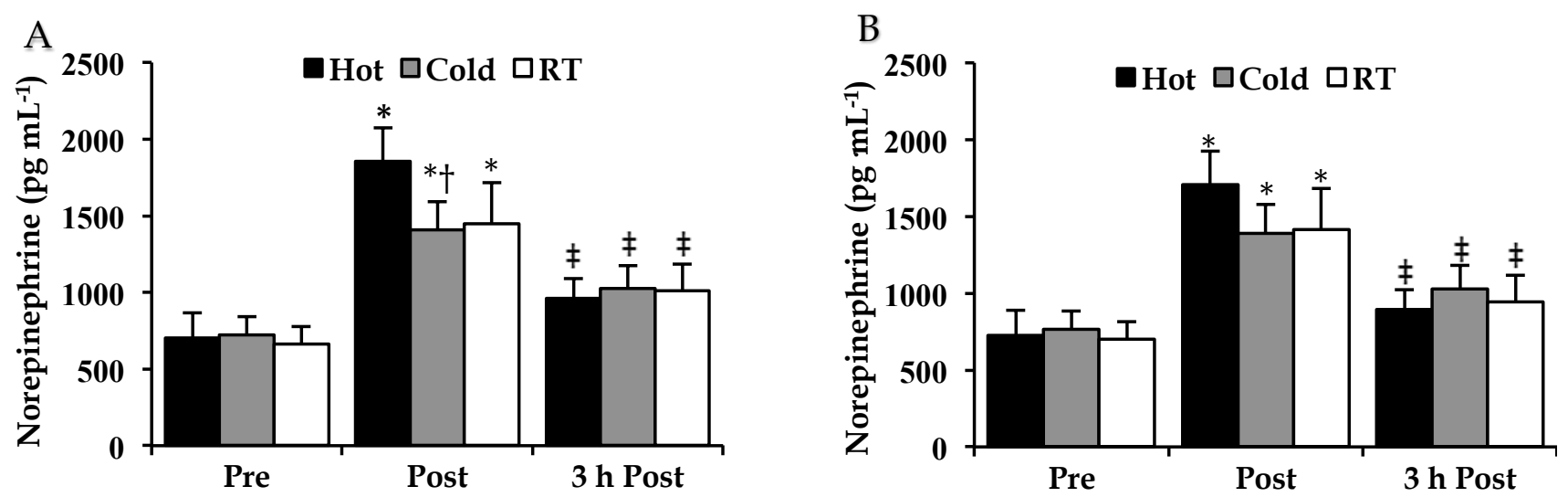

Figure 4. Norepinephrine plasma concentrations without plasma volume shift corrections (A) and after correcting for plasma volume shifts (B), ${ }^{*} p<0.05$ from pre-exercise, $\uparrow \mathrm{p}<0.05$ from hot, $\ddagger \mathrm{p}<0.05$ from post-exercise. Data are mean \pm SE.

Norepinephrine plasma concentrations increased from pre-exercise to post-exercise $(\mathrm{p}<0.01)$ and decreased from post-exercise to $3 \mathrm{~h}$ post-exercise $(\mathrm{p}<0.01)$ regardless of condition (Figure $4 \mathrm{~A})$. Post-exercise norepinephrine was significantly lower in the cold compared to hot $(\mathrm{p}=$ 0.047, Figure 4A). When norepinephrine concentrations were corrected for plasma volume shifts the same pattern of increasing from pre to post exercise and decreasing from post- 
exercise to $3 \mathrm{~h}$ post exercise in each condition was observed $(\mathrm{p}<0.05)$. However, the difference between hot and cold at the post-exercise time-point was not evident $(p=0.099$, Figure 4B).

No significant differences in FNDC5 mRNA were observed between the hot, cold, and RT trials for any time points $(\mathrm{p}>0.05)$. Additionally, no differences were detected in FNDC5 mRNA between pre-, post-, and $3 \mathrm{~h}$ post-exercise (Figure 5).

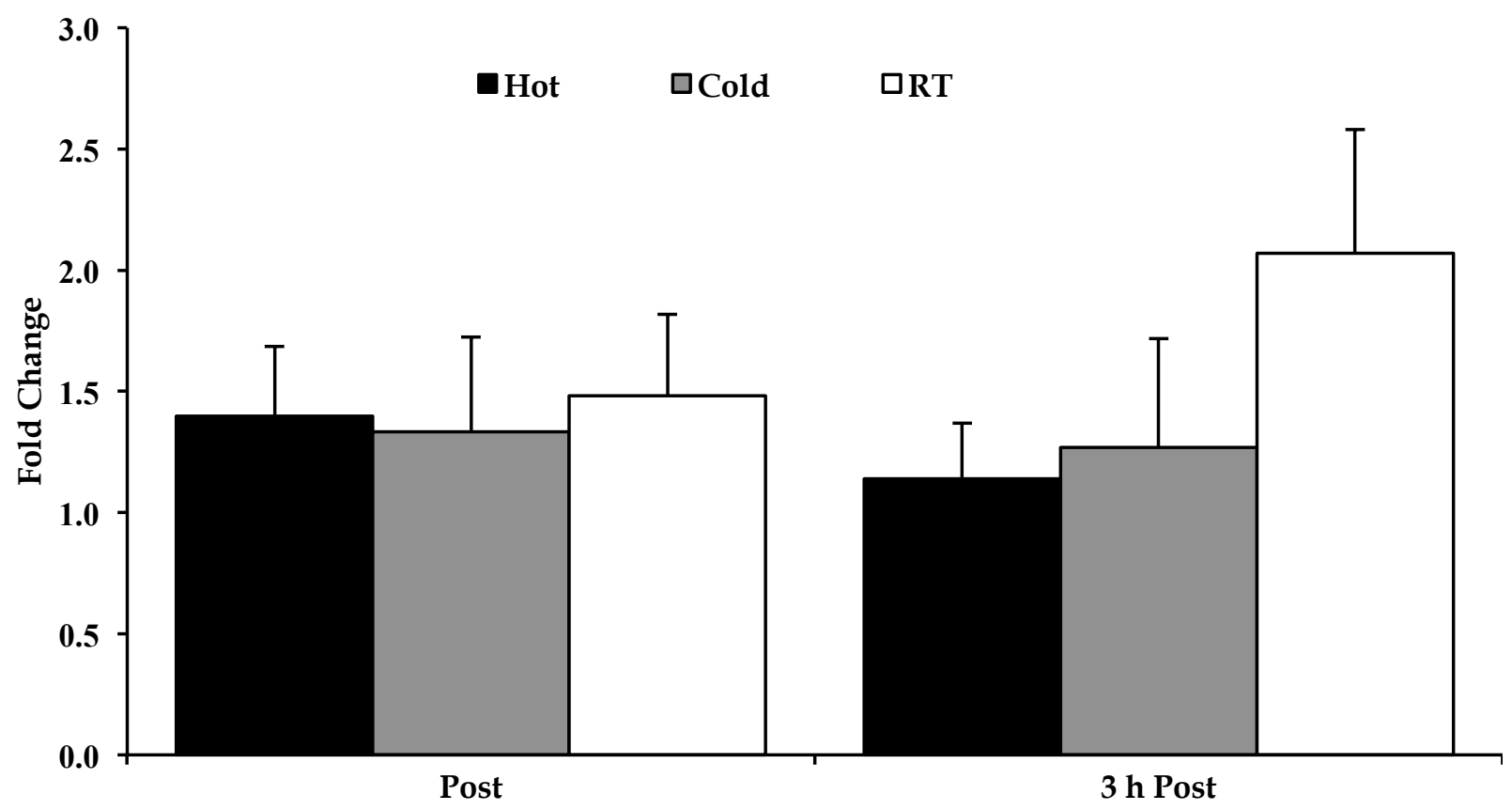

Figure 5. Gene expression of FNDC5 immediately post-exercise and $3 \mathrm{~h}$ post-exercise normalized to pre-exercise. Data are mean \pm SE.

\section{DISCUSSION}

Exercise has the capability to improve muscular and metabolic health through the regulation of key genes, hormones, and myokines. The ability to maximize the effectiveness of a given exercise bout with novel endurance exercise protocols, coupled with environmental temperature exposure interventions may help to mitigate metabolic dysfunction and obesity. The viability of the browned WAT to aid in protection against metabolic dysfunction and obesity has not yet been completely clarified in a human model. However, animal studies suggest a major role of BAT in overall health (36). Thus, we aimed to determine the impact of endurance exercise in different environmental temperatures on muscle derived signals, specifically FDNC5 and irisin, as well as plasma epinephrine and norepinephrine.

Based on previous research that indicates potential increases in irisin due to exercise absent of cold exposure $(2,4,5,16,20,33,40)$, and increases in irisin via a cold exposure absent of exercise $(2,20,27)$, it was hypothesized that exercise in a cold environment would elicit greater circulating irisin and skeletal muscle gene expression of FNDC5 when compared to exercise in 
a room temperature and hot environment. Contrary to the hypothesis, the main finding of this investigation was irisin plasma concentrations and FNDC5 mRNA were not different between hot, cold, and room temperature conditions. However, irisin plasma concentrations did tend to increase (non-significant) from pre- to post-exercise and decrease significantly from postexercise to $3 \mathrm{~h}$ post-exercise regardless of temperature when a shift in plasma volume was not considered. However, after correcting for plasma volume shifts, no differences in irisin were observed. Furthermore, we did not observe a change in FNDC5 skeletal muscle mRNA with exercise or temperature.

This study was the first to determine the effects of a hot, cold, and RT environmental condition during exercise on circulating irisin and skeletal muscle FNDC5 mRNA in humans. Previous research suggests a disassociation between energy expenditure and irisin, which requires supplementary cold-specific signals in order to produce irisin (20). During rest, subjects exposed to cold temperatures, that did not cause a change in core temperature, did show an increase in irisin (20). This previous research suggests that cold induced signals independent of a change in core body temperature may be responsible for the irisin response. In the current study, core body temperature increased during exercise in all conditions with only moderate, short-lived further elevation in the hot trial compared to cold and RT. This relative increase in core body temperature regardless of trial may have prevented us from observing changes in circulating irisin or FNDC5 mRNA. The cold stimulation during the present study was not enough to prevent a rise core body temperature with the imposed exercise. The extent to which an increase in core body temperature may prevent a rise in irisin or FNDC5 is unknown. However, exercise alone has been shown to increase irisin (2).

Norepinephrine and epinephrine up regulate PGC-1 $\alpha$ mRNA (11), and subsequently the downstream factors, specifically FNDC5 (2). Elevated levels of epinephrine and norepinephrine are observed after exercise in all exercise conditions with greater increases in hot environments (17). This study corroborates that epinephrine and norepinephrine increase during exercise, more so in the heat than at RT and cold environment. However, increases in these catecholamines due to temperature differences did not stimulate a differential increases in circulating irisin or FNDC5 mRNA.

Exercise may mimic shivering thermogenesis and produce irisin and FNDC5 mRNA in an analogous manner $(2,20)$. Specifically, cycling at room temperature for $45 \mathrm{~min}$ at $70 \%$ of $\mathrm{VO}_{2 \max }$ produces an acute increase in irisin in healthy, physically inactive men (age 45-60) (26). However, this study did not correct for plasma volume shifts known to occur with exercise, and the impact that this correction may have made is unknown. The current study, using young college-aged, active participants and at a lower exercise intensity, did not detected any increases in irisin (uncorrected for plasma volume shifts). Only one other study has corrected irisin plasma concentrations taking into account plasma volume shifts. This study observed an increase in irisin plasma concentrations at $54 \mathrm{~min}$ of a $90 \mathrm{~min}$ treadmill exercise bout at $60 \%$ of $\mathrm{VO}_{2 \max }$ in healthy, active males and females (18). However, at 90 min of treadmill running, irisin plasma levels returned to baseline levels (18). No differences were observed at the $3 \mathrm{~h}$ post-exercise after correcting for plasma volume shifts. Thus, the timing of irisin 
measurements relative to exercise duration, the correction of irisin concentrations relative to plasma volume, and exercise intensity may all contribute to differences between studies. Increased relative intensity of exercise has been shown to increase the production of irisin (40). However, during the present study the relative intensity $\left(\mathrm{VO}_{2}\right)$ was greater in the hot compared to the RT $(8.3 \%)$ and cold $(12.4 \%)$ conditions. It would be reasonable to speculate that irisin would be elevated in the hot condition. Thus, the relative intensity of the exercise bout may not be a major factor for increasing irisin when absolute intensity (watts) is held constant. Several of the studies discussed did not account for plasma volume shifts that occur during endurance exercise $(2,15,20,33)$. It is not necessary to correct for plasma volume shifts in rodents due to the inability of the rodent to sweat. In humans irisin concentrations could be inflated due to plasma lost via sweating and other mechanisms of fluid loss. Therefore plasma volume shifts should be taken into consideration. However, increasing the concentration of irisin in plasma without increasing the total amount of irisin may potentially increase the potential interaction with its receptors. We report here both corrected and uncorrected irisin concentrations so that future research can consider this impact.

We did not observe a difference in FNDC5 mRNA between trials or with exercise. Similarly, obese rat models $(3,30)$ and other human studies $(38)$ have not observed a change in FNDC5 mRNA during aerobic exercise. With 10 weeks of chronic endurance training, an increase in basal levels of FNDC5 mRNA was observed but this increase did not lead to an increase in plasma irisin. Furthermore, aerobic endurance training and strength endurance training lasting for 26 weeks showed no changes in plasma irisin or FNDC5 mRNA (13). With chronic sprint training, a reduction in plasma levels of irisin were observed after eight weeks (16). The discrepancies in FNDC5 and irisin responses to exercise suggest a complex interaction of factors and that mechanisms other than FNDC5 transcription may regulate irisin release during the early and late responses to acute and chronic exercise in humans.

Age and muscle mass are primary predicators of irisin and FNDC5 levels, a positive correlation with muscle mass and a negative correlation with age (27). Therefore, increased muscle mass should produce an increase in irisin response during exercise and cold-exposure. However, the participants in this study were college-aged males, relatively lean, and fit and no increases in irisin or FNDC5 mRNA were observed during exercise and cold exposure contrary to previous research. In similarly fit individuals, based on BMI and $\mathrm{VO}_{2 \text { max }}$, and age (18-30 years) increases in circulating irisin were observed immediately after a treadmill $\mathrm{VO}_{2 \max }$ test (7). Physically inactive, individuals, age 45-60, have also shown increases in irisin levels during cycling exercise (25). On the other end on the spectrum, obese and insulin resistant individuals have a decreased amount of irisin (23). Therefore differing fitness levels, age, and muscle mass may be potential explanations for the total amount of irisin cleaved from FNDC5 proteins (2).

The variations observed in irisin concentrations and FNDC5 mRNA expression between studies and subject populations appear to illustrate that exercise may not regulate FNDC5 mRNA expression and irisin production in humans (29). Further research is needed in order to understand human irisin production and FNDC5 mRNA expression. In conclusion, the current 
data indicate that in humans, circulating irisin and skeletal muscle FNDC5 mRNA expression post-exercise and $3 \mathrm{~h}$ post-exercise are unaffected by exposure to different environmental temperatures despite some previous evidence of independent effects of exercise and cold exposure.

\section{ACKNOWLEDGEMENTS}

This publication was made possible by grants from the University of Nebraska at Omaha Graduate Research and Creative Activity Grant and the National Institute for General Medical Science (NIGMS, P20GM103427), a component of the National Institutes of Health (NIH) and its contents are the sole responsibility of the authors and do not necessarily represent the official views of NIGMS or NIH.

\section{REFERENCES}

1. Bergstrom J. Muscle electrolytes in man determined by neutron activation analysis on needle biopsy specimens. Scand J Clin Lab Invest 14(Suppl 68): 1-110, 1962.

2. Boström P, Wu J, Jedrychowski MP, Korde A, Ye L, Lo JC, Rasbach KA, Boström EA, Choi JH, Long JZ. A PGC1-[agr]-dependent myokine that drives brown-fat-like development of white fat and thermogenesis. Nature 481(7382): 463-468, 2012.

3. Brenmoehl J, Albrecht E, Komolka K, Schering L, Langhammer M, Hoeflich A, Maak S. Irisin is elevated in skeletal muscle and serum of mice immediately after acute exercise. Int J Biol Sci 10(3): 338-349, 2014.

4. Busiello RA, Savarese S, Lombardi A. Mitochondrial uncoupling proteins and energy metabolism. Frontiers Physiol 6: 36, 2015.

5. Castillo-Quan JI. From white to brown fat through the PGC-1alpha-dependent myokine irisin: implications for diabetes and obesity. Dis Model Mech 5(3): 293-295, 2012.

6. Costill DL, Fink WJ. Plasma volume changes following exercise and thermal dehydration. J Appl Physiol 37(4): 521-525, 1974.

7. Daskalopoulou SS, Cooke AB, Gomez YH, Mutter AF, Filippaios A, Mesfum ET, Mantzoros CS. Plasma irisin levels progressively increase in response to increasing exercise workloads in young, healthy, active subjects. Eur J Endocrinol 171(3): 343-352, 2014.

8. Erickson HP. Irisin and FNDC5 in retrospect: an exercise hormone or a transmembrane protein. Adipocyte 2.4, 289-293, 2013.

9. Febbraio MA, Lambert DL, Starkie RL, Proietto J, Hargreaves M. Effect of epinephrine on muscle glycogenolysis during exercise in trained men. J Appl Physiol (1985) 84(2): 465-470, 1998.

10. Ferrer Martínez A, Ruiz Lozano P, Chien KR. Mouse PeP: a novel peroxisomal protein linked to myoblast differentiation and development. Dev Dynamics 224(2): 154-167, 2002.

11. Fu J, Li Z, Zhang H, Mao Y, Wang A, Wang X, Zou Z, Zhang X. Molecular pathways regulating the formation of brown-like adipocytes in white adipose tissue. Diabetes Metab Res 31:433-452, 2015. 
12. Handschin C, Spiegelman BM. The role of exercise and PGC1 in inflammation and chronic disease. Nature 454(7203): 463-469, 2008.

13. Hecksteden A, Wegmann M, Steffen A, Kraushaar J, Morsch A, Ruppenthal S, Kaestner L, Meyer T. Irisin and exercise training in humans - results from a randomized controlled training trial. BMC Med 11: 235, 2013.

14. Hee Park K, Zaichenko L, Brinkoetter M, Thakkar B, Sahin-Efe A, Joung KE, Tsoukas MA, Geladari EV, Huh JY, Dincer F. Circulating irisin in relation to insulin resistance and the metabolic syndrome. J Clin Endocrinol Metab 98(12): 4899-4907, 2013.

15. Hee Park K, Zaichenko L, Brinkoetter M, Thakkar B, Sahin-Efe A, Joung KE, Tsoukas MA, Geladari EV, Huh JY, Dincer F. Circulating irisin in relation to insulin resistance and the metabolic syndrome. J Clin Endocrinol Metab 98(12): 4899-4907, 2013.

16. Huh JY, Panagiotou G, Mougios V, Brinkoetter M, Vamvini MT, Schneider BE, Mantzoros CS. FNDC5 and irisin in humans: I. Predictors of circulating concentrations in serum and plasma and II. mRNA expression and circulating concentrations in response to weight loss and exercise. Metab Clin Exp 61(12): 1725-1738, 2012.

17. Iguchi M, Littmann AE, Chang SH, Wester LA, Knipper JS, Shields RK. Heat stress and cardiovascular, hormonal, and heat shock proteins in humans. J Athl Train 47(2): 184-190, 2012.

18. Kraemer RR, Shockett P, Webb ND, Shah U, Castracane VD. A transient elevated irisin blood concentration in response to prolonged, moderate aerobic exercise in young men and women. Horm Metab Res 46(2): 150-154, 2014.

19. Lecker SH, Zavin A, Cao P, Arena R, Allsup K, Daniels KM, Joseph J, Schulze PC, Forman DE. Expression of the irisin precursor FNDC5 in skeletal muscle correlates with aerobic exercise performance in patients with heart failure. Circ Heart Fail 5(6): 812-818, 2012.

20. Lee P, Linderman JD, Smith S, Brychta RJ, Wang J, Idelson C, Perron RM, Werner CD, Phan GQ, Kammula US. Irisin and FGF21 are cold-induced endocrine activators of brown fat function in humans. Cell Metab 19(2): 302309, 2014.

21. Livak KJ, Schmittgen TD. Analysis of Relative Gene Expression Data Using Real-Time Quantitative PCR and the 2- ${ }^{-\mathrm{CT}}$ Method. Methods 25(4): 402-408, 2001.

22. Mahoney DJ, Parise G, Melov S, Safdar A, Tarnopolsky MA. Analysis of global mRNA expression in human skeletal muscle during recovery from endurance exercise. FASEB J 19(11): 1498-1500, 2005.

23. Moreno-Navarrete JM, Ortega F, Serrano M, Guerra E, Pardo G, Tinahones F, Ricart W, Fernández-Real JM. Irisin is expressed and produced by human muscle and adipose tissue in association with obesity and insulin resistance. J Clin Endocrinol Metab 98(4): E769-E778, 2013.

24. Nedergaard J, Golozoubova V, Matthias A, Asadi A, Jacobsson A, Cannon B. UCP1: the only protein able to mediate adaptive non-shivering thermogenesis and metabolic inefficiency. Biochimica et Biophysica Acta (BBA)Bioenergetics 1504(1): 82-106, 2001.

25. Norheim F, Langleite TM, Hjorth M, Holen T, Kielland A, Stadheim HK, Gulseth HL, Birkeland KI, Jensen J, Drevon CA. The effects of acute and chronic exercise on PGC 1 , irisin and browning of subcutaneous adipose tissue in humans. FEBS Journal 281(3): 739-749, 2014. 
26. Norheim F, Langleite TM, Hjorth M, Holen T, Kielland A, Stadheim HK, Gulseth HL, Birkeland KI, Jensen J, Drevon CA. The effects of acute and chronic exercise on PGC 1 , irisin and browning of subcutaneous adipose tissue in humans. FEBS Journal 281(3): 739-749, 2014.

27. Novelle MG, Contreras C, Romero-Picó A, López M, Diéguez C. Irisin, two years later. Int J Endocrinol 2013, 2013.

28. Pedersen BK. Muscles and their myokines. J Exp Biol 214(Pt 2): 337-346, 2011.

29. Pekkala S, Wiklund PK, Hulmi JJ, Ahtiainen JP, Horttanainen M, Pöllänen E, Mäkelä KA, Kainulainen H, Häkkinen K, Nyman K. Are skeletal muscle FNDC5 gene expression and irisin release regulated by exercise and related to health? J Physiol 591(21): 5393-5400, 2013.

30. Peterson JM, Mart R, Bond CE. Effect of obesity and exercise on the expression of the novel myokines, Myonectin and Fibronectin type III domain containing 5. PeerJ 2: e605, 2014.

31. Roca-Rivada A, Castelao C, Senin LL, Landrove MO, Baltar J, Belen Crujeiras A, Seoane LM, Casanueva FF, Pardo M. FNDC5/irisin is not only a myokine but also an adipokine. PloS One 8(4): e60563, 2013.

32. Seale P, Lazar MA. Brown fat in humans: turning up the heat on obesity. Diabetes 58(7): 1482-1484, 2009.

33. Shan T, Liang X, Bi P, Kuang S. Myostatin knockout drives browning of white adipose tissue through activating the AMPK-PGC1alpha-Fndc5 pathway in muscle. FASEB J 27(5): 1981-1989, 2013.

34. Siri WE. Body composition from fluid spaces and density: analysis of methods. 1961. Nutrition 9(5): 480-91; discussion 480, 492, 1993.

35. Slivka D, Dumke C, Tucker T, Cuddy J, Ruby B. Human mRNA response to exercise and temperature. Int J Sports Med 33(02): 94-100, 2012.

36. Stanford KI, Middelbeek RJ, Townsend KL, An D, Nygaard EB, Hitchcox KM, Markan KR, Nakano K, Hirshman MF, Tseng YH, Goodyear LJ. Brown adipose tissue regulates glucose homeostasis and insulin sensitivity. J Clin Invest 123(1): 215-223, 2013.

37. Thomas TR, Etheridge GL. Hydrostatic weighing at residual volume and functional residual capacity. J Appl Physiol Respir Environ Exerc Physiol 49(1): 157-159, 1980.

38. Timmons JA, Baar K, Davidsen PK, Atherton PJ. Is irisin a human exercise gene? Nature 488(7413): E9-E10, 2012.

39. Tseng Y, Cypess AM, Kahn CR. Cellular bioenergetics as a target for obesity therapy. Nature Rev Drug Discovery 9(6): 465-482, 2010.

40. Tsuchiya Y, Ando D, Goto K, Kiuchi M, Yamakita M, Koyama K. High-intensity exercise causes greater irisin response compared with low-intensity exercise under similar energy consumption. Tohoku J Exp Med 233(2): 135-140, 2014.

41. Uldry M, Yang W, St-Pierre J, Lin J, Seale P, Spiegelman BM. Complementary action of the PGC-1 coactivators in mitochondrial biogenesis and brown fat differentiation. Cell metabolism 3(5): 333-341, 2006.

42. World Medical Association. Declaration of Helsinki, ethical principles for medical research involving human subjects. 52nd WMA General Assembly, Edinburgh, Scotland, 2000. 
Int J Exerc Sci 10(5): 666-680, 2017

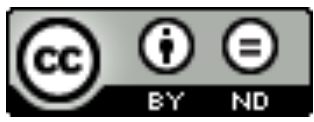

International Journal of Exercise Science ( 680 